\title{
SIMULTANEOUS DETERMINATION OF CEFTRIAXONE SODIUM AND STATIN DRUGS IN PHARMACEUTICAL FORMULATIONS AND HUMAN SERUM BY RP-HPLC
}

\author{
NAJMA SULTANA', M. SAEED ARAYNE2 AND WASEEM SHAHZAD \\ ${ }^{I}$ Research Institute of Pharmaceutical Sciences, Department of Pharmaceutical Chemistry Faculty of Pharmacy \\ University of Karachi, Karachi-75270, Pakistan. \\ ${ }^{2}$ Department of Chemistry, University of Karachi, University of Karachi.
}

(Received: June 30, 2009 - Accepted: January 10, 2010)

\begin{abstract}
An accurate, sensitive and least time consuming reverse phase high performance liquid chromatographic (RP-HPLC) method for the estimation of ceftriaxone in the presence of statin drugs in formulation and human serum has been developed and validated. Chromatographic separation was conducted on prepacked Purospher Star, C18 (5 mm, $250 \times 4.6 \mathrm{~mm})$ column at room temperature using methanol:water:acetonitrile $(70: 15: 20 \mathrm{v} / \mathrm{v} / \mathrm{v})$ as a mobile phase, $\mathrm{pH}$ adjusted at 2.8 with ortho-phosphoric acid and at a flow rate of $1.0 \mathrm{~mL} /$ minute, while UV detection was performed at $240 \mathrm{~nm}$. The results obtained showed a good agreement with the declared content. The method shows good linearity in the range of $2.5-25 \mu \mathrm{g} / \mathrm{mL}$ with a correlation coefficient $0.9966-0.9998$ (inter-and intra-day $\mathrm{RSD}<2 \%$ ). The limit of detection and quantification for ceftriaxone and statins in pharmaceutical formulation and serum were in the range $0.124-1.006 \mu \mathrm{g} / \mathrm{mL}$. Analytical recovery was $>98.72 \%$. The proposed method may be used for the quantitative analysis of commonly administered statin's i.e. simvastatin, rosuvastatin atorvastatin and pravastatin alone or in combination with ceftriaxone from raw materials, dosage formulations and in serum. The established HPLC method is rapid, accurate and selective, because of its sensitivity and reproducibility.
\end{abstract}

Keywords: ceftriaxone, statin's, simvastatin, rosuvastatin, atorvastatin, pravastatin and RP-HPLC.

\section{INTRODUCTION}

Ceftriaxone (fig.1) is a (6R,7R)-7-\{2-(2-amino-4-thiazolyl)-(Z)-2[methoxyiminuteo-acetamido]-3 $\{[(2,5$-dihydro-6-hydroxy-2-methyl-5-oxoas-triazin-3-yl)thio]methyl\}-8-oxo-5-thia-1-azobicyclo $[4,2,0]$ oct-2-ene-2carboxylic acid, in the hydrated disodium salt and contains approximately $83 \mathrm{mg}$ of sodium per gram of ceftriaxone. It is a third generation parentral cephalosporin with a relatively long half life which is stable to $\beta$-lactamases particularly those produced by Gram-negative organisms [1- 6]. It has excellent anti Gram-negative activity. It contains a highly acidic heterocyclic system on the 3-thiomethyl group, this unusual ring system is believed to confer the unique pharmacokinetic properties to this agent. It kills bacteria by interfering in the synthesis of the cell wall. Ceftriaxone has been effective in treating infections due to other 'difficult' organisms such as multi drug-resistant Enterobacteriaceae, [7- 12].<smiles>CO/N=C(\C(=O)N[C@H]1C(=O)N2C(C(=O)O)=C(CSc3nc(=O)c(=O)[nH]n3C)CS[C@H]12)c1csc(N)n1</smiles>

Fig. 1: Ceftriaxone

Statin's are a group of drugs used primarily in lowering cholestero. They are generally capable of lowering cholesterol levels by 20 to 60 percent. The discovery of HMG-CoA (3-hydroxy-3-methylglutaryl-CoA) reductase inhibitors, called statins, was a breakthrough in the prevention of hypercholesterolemia and related diseases.[13-16]

Cholesterol plays an important role in the everyday functioning of the body. Unfortunately, it can also have a negative effect, contributing to the development of atherosclerosis. These plaques can block the arteries, cutting off blood flow, or rupturing and causing a clot that increases blockage. The results of such blockages are very serious and can include angina, claudication, heart attack and stroke [17-19].

Available in tablet or capsule form, statin's are usually taken with dinner or at bedtime. Results are typically evident after a period of four to six weeks of use. Medications in this group are usually easy to tolerate and cause few, if any, side effects [20].

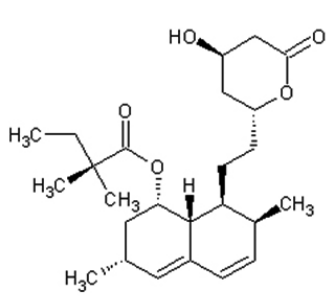

simvastatin

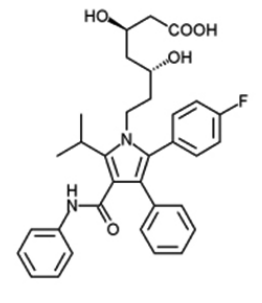

atorvastatin



rosuvastatin<smiles>CC[C@H](C)C(=O)O[C@H]1C[C@H](O)C=C2C=C[C@H](O)[C@H](CC[C@H](O)C[C@H](O)CC(C)=O)[C@H]21</smiles>

pravastatin

Fig. 2

The statins differ with respect to their ring structure and substituents. These differences in structure affect the pharmacological properties of the statins. Statins have sometimes been grouped into two groups of statins according to their structure. Statins that belong to type 1 are pravastatin and simvastatin. Statins that are fully synthetic and have larger groups linked to the HMG-like moiety are often referred to as type 2 statins. Statins that belong to this group are atorvastatin and rosuvastatin [21-23].

The scope of study was to investigate the possible effect of these statin's on emergency treatment with ceftriaxone. The present method was actually developed in our laboratories for drug-drug interaction studies between ceftriaxone and statin's in order to study the effect of these on the availability of ceftriaxone. Such a method was needed for co-administration of both drugs is possible in multiple drug therapy and there is no such method reported in the literature for the simultaneous determination of ceftriaxone and statin's. Furthermore, we report a specific and sensitive HPLC method for the simultaneous determination of ceftriaxone and statin's in pharmaceutical preparations and human serum.

There are a number of liquid chromatographic methods reported in the literature for the individual assays of ceftriaxone in aqueous and biological sample [24-29]. Owens achieved chromatographic separation by using cetyltrimethylammonium bromide $(0.01 \mathrm{M})$ as ion-pairing agent. The mobile phase consisted of methanol-acetonitrile-phosphate buffer, pH 7.4 (20:20:60, $\mathrm{v} / \mathrm{v} / \mathrm{v}$ ) but it was a difficult mobile phase [24]. Another HPLC method for the determination of ceftriaxone has been developed by Eric [25]. Chromatogram evaluation in the reflectance/absorbance mode. Traument [26] developed a 
method for determination of ceftriaxone in plasma, urine and bile by means of ion-pair reversed phase chromatography using phosphate buffer of $\mathrm{pH} 8$ at $254 \mathrm{~nm}$. One method for the analysis of ceftriaxone in clinical microbiology in the biological fluids has been developed by the Knoeller et al,[27]. Nahata [28], Jehl and Birckel [29] also developed a method using phosphate buffer of pH 7 as a mobile phase. All of these methods consisted buffers combination as mobile phase. Several methods also reported for determination of Statin's [30- 34].

However, no method for simultaneous determination of Statin's and ceftriaxone in active and in dosage formulations has been studied so far. On this basis, it became apparent to develop and validate for the first time a simultaneous method for the estimation of these drugs in bulk material, dosage formulations and in human serum using RP-HPLC. Several problems were resolved in the simultaneous determination of compounds investigated. The first was the selection of separation conditions to ensure efficient extraction of the drugs and from human serum with minimum interference from serum endogenous compounds. The second was the choice of proper chromatographic conditions to obtain separation of all components from the endogenous compounds. Thirdly, the method had to be sufficiently sensitive to measure concentrations of the investigated drugs in serum within their therapeutic range. The present work describes a simple RP-LC method for the simultaneous determination of ceftriaxone, Simvastatin, Rosuvastatin, Atorvastatin, and Pravastatin bulk raw materials, dosage formulations and in human serum.

\section{EXPERIMENTAL}

\section{Instrumentation and analytical conditions}

Two identical LC systems were used for the separation in two different labs: Shimadzu HPLC system equipped with LC-10 AT VP pump, DGU-14 $\mathrm{AM}$ on-line degasser, Rheodyne manual injector fitted with a $20 \mu \mathrm{L}$ loop, column and SPD-10 A VP UV-VIS detector and a Purospher star C18 $(5 \mu \mathrm{m}$, $25 \times 0.46 \mathrm{~cm})$ column separation was utilized. Chromatographic system was integrated via Shimadzu model CBM-102 Communication Bus Module to P-IV computer. Shimadzu CLASS-GC software (Version 5.03) was used for data acquisition and mathematical calculations.

\section{Materials and reagents}

HPLC grade acetonitrile, methanol and phosphoric acid were obtained from Merck Germany. Ceftriaxone (bestrix Injection) was a gift from Pharmevo Pvt Ltd. The statins used were simvastatin (limitrol ${ }^{\mathbb{1}} 10 \mathrm{mg}$ ), rosuvastatin (x-plended $5 \mathrm{mg}$ ), atorvastatin (derot ${ }^{\circledR} 10 \mathrm{mg}$ ) of Pharmevo (Pvt) Ltd and pravastatin (pravachol ${ }^{\circledR} 20 \mathrm{mg}$ ) of Bristol Myer Squibb which were purchased from the local pharmacy. All these drugs had an expiry of not less than one year at the time of study.

\section{Stock and working solution preparation}

Stock standard solutions of $100 \mu \mathrm{g} / \mathrm{mL}$ of ceftriaxone, simvastatin, atorvastatin, rosuvastatin and pravastatin were prepared individually by dissolving $10 \mathrm{mg}$ of each in $100 \mathrm{~mL}$ volumetric flask using mobile phase as a diluent. Working solutions were also prepared separately by diluting from the standard solution to obtain concentration between $2.5-25 \mu \mathrm{g} / \mathrm{mL}$. These solutions were stored at $20^{\circ} \mathrm{C}$, They were prepared once and analyzed daily for inter-day and inter-operator variations of the method and analyzed each time before drug analysis in biological samples. $20 \mu \mathrm{L}$ of these solutions were injected into LC system and chromatographed.

\section{Sample preparation}

For testing the suitability of the proposed method for the estimation of the drugs in dosage form, 20 tablets of each drug were powdered and equivalent to $10 \mathrm{mg}$ of statin's (simvastatin, atorvastatin, rosuvastatin and pravastatin) and $10 \mathrm{mg}$ of ceftriaxone were transferred to $100 \mathrm{~mL}$ volumetric flask dissolved and diluted with mobile phase. The resulting solutions were filtered through watman filter paper no. 41 and diluted to the desired concentration and analyzed for the drug content.

\section{Drug-plasma solution}

These solutions were prepared once and stored at $20^{\circ} \mathrm{C}$ for the preparation of drug serum solution. To $1 \mathrm{~mL}$ of plasma $10 \mathrm{~mL}$ of acetonitrile was added and vortexed for 1 minute and subsequently centrifuged for 10 minutes at 10,000 rpm. Supernatant was filtered through a 0.45 -micron pore size membrane filter. Serum obtained was added to the above solutions to produce the desired concentrations and stored at $20^{\circ} \mathrm{C}$. In order to evaluate linearity in serum five concentration levels ranging from $2.5-25 \mu \mathrm{g} / \mathrm{mL}$ were prepared and linearity and $\%$ R.S.D values were evaluated.

\section{RESULTS AND DISCUSSION}

This work was designed to develop an isocratic method based on RPLC separation for statin's and ceftriaxone assay in pharmaceutical dosage formulations, bulk material as well as in human serum. The goal of this study was to develop a rapid, more accurate, precise, reliable, least time consuming HPLC method for the five drugs individually as well as simultaneously. This analytical method was developed taking in account the therapeutic and overdose concentration range, has been validated and holds well for the determination of drugs in raw materials, dosage formulations and especially in human serum.

\section{Method optimization and chromatographic conditions}

To optimize the operating conditions for isocratic RP-LC detection of all analytes, a number of parameters such as the mobile phase composition, $\mathrm{pH}$ and the flow rate were varied. Various ratios $(80: 20,70: 30,60: 40 \mathrm{v} / \mathrm{v})$ of methanol: water was tested as starting solvent for system suitability study. The variation in the mobile phase leads to considerable changes in the chromatographic parameters, like peak symmetry, capacity factor and retention time. The $\mathrm{pH}$ effect showed that optimized conditions are reached when the $\mathrm{pH}$ value is 2.8 , producing well resolved and sharp peaks for all drugs assayed. Henceforth, in the present method $\mathrm{pH}$ adjusted to 2.8 using wavelength 240 $\mathrm{nm}$ (isosbestic point). However, the peak shape and resolution were found to be good when the mobile phase comprising of the methanol: water: acetonitrile $\mathrm{pH}$ adjusted to 2.8 with phosphoric acid was used in the ratio of $(70: 15: 20$ $\mathrm{v} / \mathrm{v} / \mathrm{v}$ ) at a flow rate of $1 \mathrm{~mL} / \mathrm{min}$ (filtered through a 0.45 micron filter). For simultaneous determination of ceftriaxone with statin's drugs, simvastatin, rosuvastatin, atorvastatin and pravastatin in individual drug solutions were injected into the column at the concentration of $100 \mu \mathrm{gmL}^{-1}$ and both elution pattern and resolution parameters were studied. System suitability data given in table 2. Type 1 statin's and type 2 statin's run separately with ceftriaxone due to overlapping of pravastatin and rosuvastatin. (Figure 3 and 6).

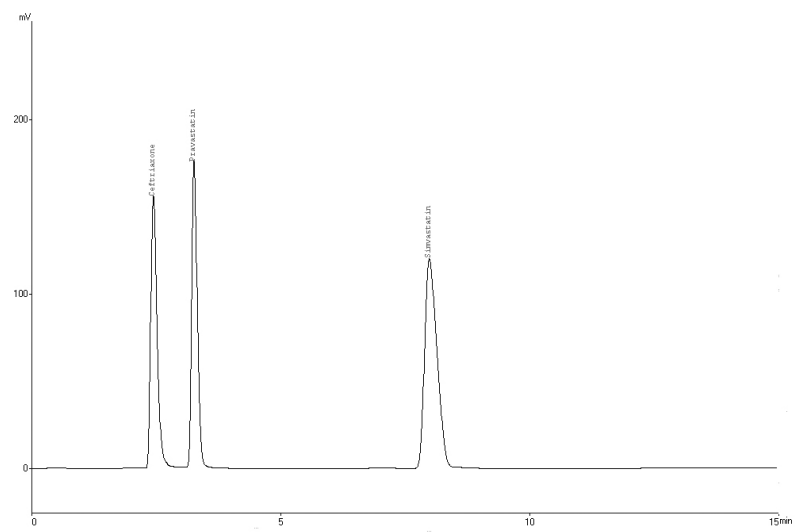

Figure 3. A representative chromatogram of ceftriaxone, Pravastatin and Simvastatin in raw material.

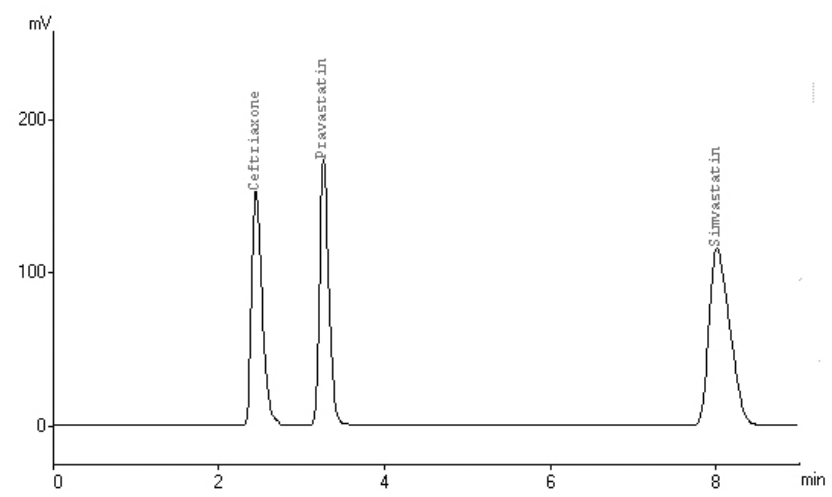

Figure 4. A representative chromatogram of ceftriaxone, pravastatin and simvastatin in serum 


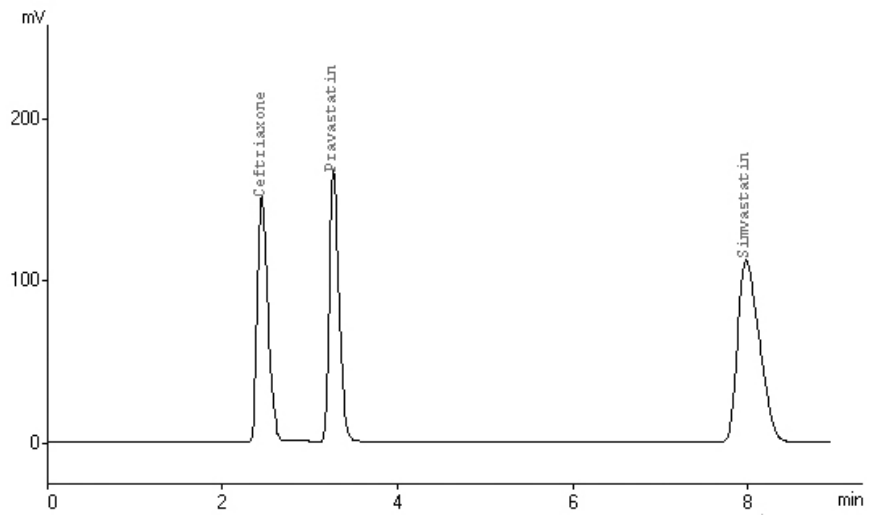

Figure 5. A representative chromatogram of ceftriaxone, pravastatin and simvastatin in formulation



Figure 6. A representative chromatogram of ceftriaxone, rosuvastatin and atorvastatin in raw material.

\section{Wavelength selection}

In addition, the UV spectra of individual drugs were recorded in the wavelength range from 200 to $400 \mathrm{~nm}$ and compared. The choice to use a common wavelength set at $240 \mathrm{~nm}$ was considered satisfactory, permitting the detection of all drugs with adequate sensitivity.

\section{Method validation}

For validation of analytical methods, the guidelines of the International Conference on the Harmonization of Technical Requirements for the Registration of Pharmaceuticals for Human Use (ICH) [35] and USP [4] have recommended the accomplishment of accuracy tests, precision, specificity, linearity and robustness of the method.

\section{System suitability}

The system suitability studies were carried out as specified by ICH [35] by analyzing the symmetry of the ceftriaxone and statin's peaks (symmetry factor $<2$ ), theoretical plates of the column $>3,000$

\section{Accuracy and precision}

Inter and intra-day precision and accuracy of the method were evaluated at different independent concentrations by adding known quantities of the analytes to the drug product (spiking method). The results of accuracy (table 3 - 5) revealed that the method was accurate for all above purposes. The method passed the test for repeatability (system suitability test table 2) as determined by $\%$ RSD of the area of the peaks of six replicate injections at $100 \%$ test concentration.



Figure 7. A representative chromatogram of ceftriaxone, rosuvastatin and atorvastatin in serum.

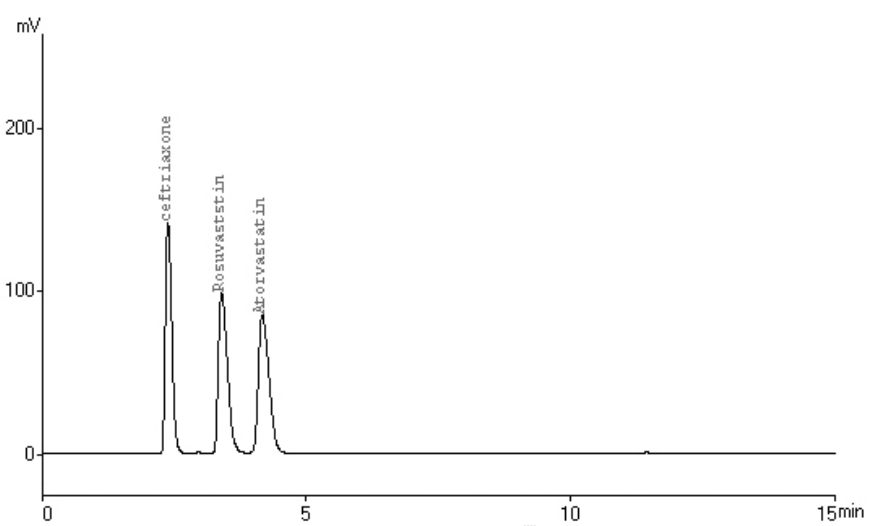

Figure 8. A representative chromatogram of ceftriaxone, rosuvastatin and atorvastatin in formulation.

\section{Specificity}

Representative chromatograms Figure. 4 and 8 were generated to show other components that could be present in the sample matrix are resolved from the parent analytes. No change was observed in the chromatogram of ceftriaxone and Statin's in the presence of common excipients. The specificity was also determined by injecting human plasma samples. Figure 5 and 7 represent human serum containing all drugs. Therefore, the proposed method is selective and specific for the drugs.

\section{Detection and quantitation limit}

The LOD and LOQ values for ceftriaxone and Statin's were determined and are presented in table 1 . The calculated LOD and LOQ values confirmed that method were sufficiently sensitive.

\section{Range and linearity}

Solutions, as described above, were injected at the interval of 4 hour on day one. This process was repeated for 4 days. Standard curves were constructed at concentrations $2.5,5,10,15,20$ and $25 \mu \mathrm{g} / \mathrm{mL}$ of all statin's and ceftriaxone. The standard calibration curves were shown to be linear in the above mentioned range in human serum. Curves were obtained by plotting the peak area against concentrations of these drugs. Linear calibration curves were generated by linear regression analysis and obtained over the respective standard concentrations ranges. The suitability of the calibration models were confirmed by back-calculating the concentrations of the calibration standards. The standard curve, slope, intercept and the correlation coefficient were determined. The regression statistics are shown in table 1.

\section{Ruggedness \& robustness}

Ruggedness of this method was evaluated in two different labs with two different instruments. Lab 1 was in the Research Institute of Pharmaceutical 
Sciences, Faculty of Pharmacy University of Karachi, while Lab 2 was in the Department of Chemistry, Faculty of Science University of Karachi. The method did not show any notable deviations in results from acceptable limits. Robustness was evaluated by slight changes in $\mathrm{pH}$ levels of mobile phase and it was found that the \%R.S.D. values did not exceed more than $2 \%$. The developed method has been applied for the determination of different drug contents in formulations. The assay results shown in, demonstrates the suitability of method. Similarly the $\%$ recoveries of drugs in presence of serum reveal the applicability of method for therapeutic purposes.

\section{CONCLUSION}

In short, our method is specific, sensitive, rapid and easy to perform for simultaneous determination of ceftriaxone and statin's (simvastatin, rosuvastatin, atorvastatin and pravastatin). The limit of quantification, small sample volume and short chromatographic time of this method makes it advantageous for adaptation to routine assay requirements and enables simultaneous determination of statin's and ceftriaxone because of good separation and resolution of the chromatographic peaks. The obtained results are in good agreement with the declared contents of dosage formulations. Results are accurate and precise and are confirmed by the statistical parameters. Reliability, rapidness, simplicity, sensitivity, economical nature, good recovery and precision of this method give it advantage over the other reported methods.

Table 1: Regression statistics

\begin{tabular}{|l|c|c|c|c|c|c|}
\hline Drugs & $\begin{array}{c}\text { Linearity } \\
\text { range }(\mu \mathrm{g} / \\
\mathrm{mL})\end{array}$ & $\begin{array}{c}\%- \\
\mathrm{RSD}\end{array}$ & $\begin{array}{c}\%- \\
\text { Recovery }\end{array}$ & $\mathrm{r}^{2}$ & LOD & LOQ \\
\hline Ceftriaxone & $2.5-25$ & 0.55 & 100.75 & 0.9966 & 0.284 & 0.860 \\
\hline Rosuvastatin & $2.5-25$ & 0.44 & 100.52 & 0.9997 & 0.172 & 0.520 \\
\hline Atorvastatin & $2.5-25$ & 0.62 & 100.70 & 0.9977 & 0.124 & 0.376 \\
\hline Pravastatin & $2.5-25$ & 1.26 & 101.39 & 0.9998 & 0.332 & 1.006 \\
\hline Simvastatin & $2.5-25$ & 1.17 & 101.25 & 0.9995 & 0.290 & 0.879 \\
\hline
\end{tabular}

Table 2: System suitability parameters.

\begin{tabular}{|l|c|c|c|c|c|}
\hline Drugs & $\begin{array}{c}\text { Ret. Time } \\
\text { (minutes) }\end{array}$ & $\mathrm{k}$ & Tailing & Resolution & $\begin{array}{c}\text { Separation } \\
\text { factor }\end{array}$ \\
\hline Ceftriaxone & 2.4 & 0 & 1.45 & & \\
\hline Pravastatin & 3.2 & 0.34 & 1.34 & 3.59 & 0 \\
\hline Rosuvastatin & 3.3 & 0.43 & 1.41 & 3.28 & 0 \\
\hline Atorvastatin & 4.0 & 0.71 & 1.46 & 1.84 & 1.68 \\
\hline Simvastatin & 8.0 & 2.29 & 1.41 & 13.51 & 6.79 \\
\hline
\end{tabular}

Table 3: Interday accuracy and precision of ceftriaxone and statin's

\begin{tabular}{|c|c|c|c|c|c|}
\hline Drugs & Conc.* & Conc.* & Conc. found & \%R.S.D. & $\%$ Recovery \\
\hline & & $\mu \mathrm{g} / \mathrm{mL}$ & $\mu \mathrm{g} / \mathrm{mL}$ & Precision & Accuracy \\
\hline \multirow{3}{*}{ Ceftriaxone } & $80 \%$ & 8 & 8.00 & 0.01 & 99.99 \\
\hline & $100 \%$ & 10 & 10.12 & 0.83 & 101.19 \\
\hline & $120 \%$ & 12 & 12.20 & 1.19 & 101.69 \\
\hline \multirow{3}{*}{ Rosuvastatin } & $80 \%$ & 8 & 8.00 & 0.04 & 99.94 \\
\hline & $100 \%$ & 10 & 10.13 & 0.88 & 101.26 \\
\hline & $120 \%$ & 12 & 12.02 & 0.13 & 100.19 \\
\hline \multirow{3}{*}{ Atorvastatin } & $80 \%$ & 8 & 7.99 & 0.13 & 99.82 \\
\hline & $100 \%$ & 10 & 10.25 & 1.75 & 102.50 \\
\hline & $120 \%$ & 12 & 12.01 & 0.08 & 100.12 \\
\hline \multirow{3}{*}{ Pravastatin } & $80 \%$ & 8 & 8.00 & 0.04 & 99.95 \\
\hline & $100 \%$ & 10 & 10.11 & 0.76 & 101.08 \\
\hline & $120 \%$ & 12 & 12.10 & 0.58 & 100.83 \\
\hline \multirow{3}{*}{ Simvastatin } & $80 \%$ & 8 & 7.99 & 0.05 & 99.93 \\
\hline & $100 \%$ & 10 & 10.10 & 0.70 & 100.99 \\
\hline & $120 \%$ & 12 & 12.07 & 0.43 & 100.61 \\
\hline
\end{tabular}

*Concentration 
Table 4: Precision and recovery of ceftriaxone and statin's in formulation.

\begin{tabular}{|c|c|c|c|c|c|}
\hline \multirow[t]{2}{*}{ Drugs } & \multirow{2}{*}{$\frac{\text { Conc. injected }}{(\mu \mathrm{g} / \mathrm{mL})}$} & \multicolumn{3}{|c|}{ Conc. found $(\mu \mathrm{g} / \mathrm{mL})$} & \multirow{2}{*}{$\begin{array}{l}\% \text { RSD } \\
\text { Mean }\end{array}$} \\
\hline & & 24hours & 48hours & 72hours & \\
\hline \multirow{3}{*}{ Ceftriaxone } & 8 & 8.01 & 7.90 & 8.04 & 0.35 \\
\hline & 10 & 10.13 & 10.20 & 10.20 & 1.13 \\
\hline & 12 & 11.68 & 11.91 & 11.95 & 0.98 \\
\hline \multirow{3}{*}{ Rosuvastatin } & 8 & 8.08 & 8.02 & 7.87 & 0.52 \\
\hline & 10 & 9.83 & 10.23 & 10.23 & 1.34 \\
\hline & 12 & 11.93 & 11.95 & 12.00 & 0.21 \\
\hline \multirow{3}{*}{ Atorvastatin } & 8 & 7.91 & 8.04 & 8.02 & 0.38 \\
\hline & 10 & 10.00 & 10.07 & 10.07 & 0.69 \\
\hline & 12 & 11.97 & 11.81 & 11.93 & 0.44 \\
\hline \multirow{3}{*}{ Pravastatin } & 8 & 8.20 & 8.05 & 8.99 & 0.46 \\
\hline & 10 & 9.76 & 9.76 & 10.18 & 0.56 \\
\hline & 12 & 12.09 & 11.47 & 12.12 & 0.26 \\
\hline \multirow{3}{*}{ Simvastatin } & 8 & 8.22 & 8.13 & 8.05 & 1.10 \\
\hline & 10 & 10.09 & 9.78 & 10.05 & 0.43 \\
\hline & 12 & 12.08 & 10.95 & 11.95 & 0.29 \\
\hline
\end{tabular}

Table 5: Precision and accuracy of ceftriaxone and statin's in human serum

\begin{tabular}{|c|c|c|c|c|c|c|}
\hline \multirow[b]{2}{*}{ Drug } & \multicolumn{3}{|c|}{ with in day } & \multicolumn{3}{|c|}{ between day } \\
\hline & Conc. $(\mu \mathrm{g} / \mathrm{mL})$ & $\begin{array}{l}\text { Amount } \\
\text { recovered }\end{array}$ & $\%$ RSD & $\begin{array}{c}\text { Amount } \\
\text { recovered }\end{array}$ & Mean \%recovery & $\begin{array}{c}\text { Mean \% } \\
\text { RSD }\end{array}$ \\
\hline \multirow{3}{*}{ Ceftriaxone } & 8 & 8.00 & 0.01 & 7.98 & 99.81 & 0.47 \\
\hline & 10 & 10.12 & 0.83 & 10.18 & 101.75 & 1.23 \\
\hline & 12 & 12.20 & 1.19 & 11.85 & 98.72 & 0.91 \\
\hline \multirow{3}{*}{ Rosuvastatin } & 8 & 8.00 & 0.04 & 7.99 & 99.88 & 0.68 \\
\hline & 10 & 10.13 & 0.88 & 10.10 & 100.96 & 1.49 \\
\hline & 12 & 12.02 & 0.13 & 11.96 & 99.69 & 0.24 \\
\hline \multirow{3}{*}{ Atorvastatin } & 8 & 7.99 & 0.13 & 7.99 & 99.86 & 0.46 \\
\hline & 10 & 10.25 & 1.75 & 10.05 & 100.48 & 0.34 \\
\hline & 12 & 12.01 & 0.08 & 11.90 & 99.21 & 0.57 \\
\hline \multirow{3}{*}{ Pravastatin } & 8 & 8.00 & 0.04 & 8.05 & 100.67 & 0.99 \\
\hline & 10 & 10.11 & 0.76 & 9.93 & 99.25 & 0.53 \\
\hline & 12 & 12.10 & 0.58 & 12.07 & 100.55 & 0.58 \\
\hline \multirow{3}{*}{ Simvastatin } & 8 & 7.99 & 0.05 & 8.11 & 101.40 & 0.98 \\
\hline & 10 & 10.10 & 0.70 & 10.07 & 100.69 & 0.48 \\
\hline & 12 & 12.07 & 0.43 & 12.03 & 100.23 & 0.57 \\
\hline
\end{tabular}




\section{REFERENCES}

1. Martindale: The Complete Drug Reference Pharmaceutical Press London. 33th ed, 176-177 2002.

2. H.P. Rang, and M.M. Dale, Pharmacology, Churchill Livingstone, ELBS 2nd edition 815, 1993.

3. British Pharmacopoeia, London-The stationary office 2009 .

4. USP NF, United state Pharmacopeial convention Inc, 2009.

5. Physicians Desk Reference, American Academy of Physician Assistants. 51 th edition, 833, 1997.

6. European Pharmacopoeia Strasbourg: Council of Europe, 2002.

7. P. Moser, A. Sallmann and I. Wiesenberg, J. Med. Chem. 33, 2358-2368 (1990).

8. Alfonso, R.G, Remington: The Science and Practice of Pharmacy, Mack Publishing Company, Easton, Pennsylvania, 19th edition. 2, 1211-1215, 1995.

9. Colin D, Therapeutic Drug: Churchill Livingstone, Division of Longman group UK. Ltd. 2nd edition. 88- 89, 1999.

10. Alfred, G. and Gilman, L. S. Goodman and Gilman's. The Pharmacological Basis of Therapeutics: McGraw-Hill, New York, 9th ed, 617-658, 1996.

11. Bertram, G.K. Basic and Clinical Pharmacology: Appleton and Lange. Stamford, Connecticut. 7th edition. 579-588, 1998.

12. D.H. Richards, R.C. Hell, R.V. Brogden, T.M. Speight, G. S. Avery. Ceftriaxone: a review of its antibacterial activity, pharmacological properties and therapeutic use. Drugs, 27, 469-527, (1984).

13. J.L. Goldstein and M.S. Brown "Regulation of the mevalonate pathway". Nature, 343, 425-430 (1990)

14. J.A. Tobert. "The history of the HMG-CoA reductase inhibitors". Nat Rev Drug Discov, 2, 517-526 (2003).

15. P.H. Jones, M.H. Davidson, E.A. Stein, H.E. Bays, J.M. McKenney, E. Miller, V. A. Cain and J.W. Blasetto "Comparison of the efficacy and safety of rosuvastatin versus atorvastatin, simvastatin, and pravastatin across doses (STELLAR* Trial)". Am. J. Cardiol. 92, 152-160, (2003).

16. M.J. Caslake, G. Stewart, S.P. Day, E. Daly, F. McTaggart, M.J. Chapman, P. Durrington, P. Laggner, M. Mackness, J. Pears and C.J. Packard "Phenotype-dependent and independent actions of rosuvastatin on atherogenic lipoprotein subfractions in hyperlipidaemia". Atherosclerosis, 171, 245-253, (2003).

17. D. McTavish, E.M. Sorkin. Pravastatin: a review of its pharmacological properties and therapeutic potential in hypercholesterolaemia. Drugs, 42, 65-89, (1991).

18. P.H. Chong, B.T. Yim. Rosuvastatin for the treatment of patients with hypercholesterolemia. Ann Pharmacother, 36, 93-101, (2002).

19. J.M. McKenney. Efficacy and safety of rosuvastatin in treatment of dyslipidemia. Am J Health-Syst Pharm, 62, 1033-47, (2005).

20. P.H. Chong, Lack of Therapeutic Interchangeability of HMG-CoA Reductase Inhibitors. Ann Pharmacother., 36, 1907-1917, (2002).

21. G.L. Plosker, D. McTavish, "Simvastatin: a reappraisal of its pharmacology and therapeutic efficacy in hypercholesterolaemia". Drugs, 50, 334-63 (1995).
22. G. Schectman, J. Hiatt, "Dose-response characteristics of cholesterollowering drug therapies: implications for treatment". Ann. Intern. Med., 125, 990-1000 (1996).

23. C.M. White, "Pharmacological effects of HMG CoA reductase inhibitors other than lipoprotein modulation". J. Clin. Pharmacol., 39, 111-18 (1999).

24. H.M. Owens, C.J. Destache, A.K. Dash. Journal of chromatography B Biomedical sciences and application, 728, 97-105, (1999).

25. S. Eric-Jovonovic, D. Agbaba, D. Zivanov-Stakic, S. Vladimirov, J. Pharm. Biomed. Anal., 18, 893-898, (1998).

26. K.H. Trautment, P. Haefelfinger. Journal High Res . Chromato., 4, 54-59, (1981).

27. J. Knoeller, W. Koenig, W. Schoenfeld, K. D. Bremm, M. Koeller, J. Chromatogr., 427, 257-267, (1988).

28. M.C. Nahata, Measurment of ceftriaxone in peritoneal dialysis solution by HPLC J. Liq. Chromatogr. 14, 179-185, (1991).

29. F. Jehl, P. Brickel, H. Monteil, Journal Chromato. 413, 109-119 (1987).

30. M.P. Khalid, M. Muzeeb, S.S. Jafar, Basha, D. Shashikumar, M. Ramesh and N. R. Srinivas, "Analysis of five HMG-CoA reductase inhibitors - atorvastatin, lovastatin, pravastatin, rosuvastatin and simvastatin: pharmacological, pharmacokinetic and analytical overview and development of a new method for use in pharmaceutical formulations analysis and in vitro metabolism studies". Biomedical Chromatography, 20 (3), $282-293,(2005)$

31. V. Shivva, R.S. Nikhil, R.K. Thammera, C.A. Menon, M. Ramesh, N.R. Srinivas, "Simultaneous quantitation of rosuvastatin and gemfibrozil in human plasma by high-performance liquid chromatography and its application to a pharmacokinetic study". Biomed. Chromatogr., 20 (11), 1252-1259, (2006).

32. R.K. Thammera, R.S. Nikhil, K.K. Pasikanti, C.A. Menon, P.K. Venkata, M. Ramesh and N.R. Srinivas, "Determination of rosuvastatin in rat plasma by HPLC: validation and its application to pharmacokinetic studies". Biomed. Chromatogr., 20 (9), 881-887, (2006).

33. K.T. Ravi, R.K. Raja, M. Ramesh, N.R. Srinivas, "Simultaneous determination of rosuvastatin and fenofibric acid in human plasma by LC-MS/MS with electrospray ionization: assay development, validation and application to a clinical study". J Pharm Biomed Anal., 39, 661-669, (2005).

34. V. Bořek-Dohalský, J. Huclová, B. Barrett, B. Němec, I. Ulč and I. Jelínek Validated HPLC-MS-MS method for simultaneous determination of atorvastatin and 2-hydroxyatorvastatin in human plasma. Journal of Analytical and Bioanalytical Chemistry, 386, 275-285, (2006).

35. International Conference on the Harmonization of Technical Requirements for the Registration of Pharmaceuticals for Human Use (ICH) Q2B 1996 Validation of Analytical Procedures, Methodology, 1996. 